\section{EPIGENETICS}

Not all Dollies are the same

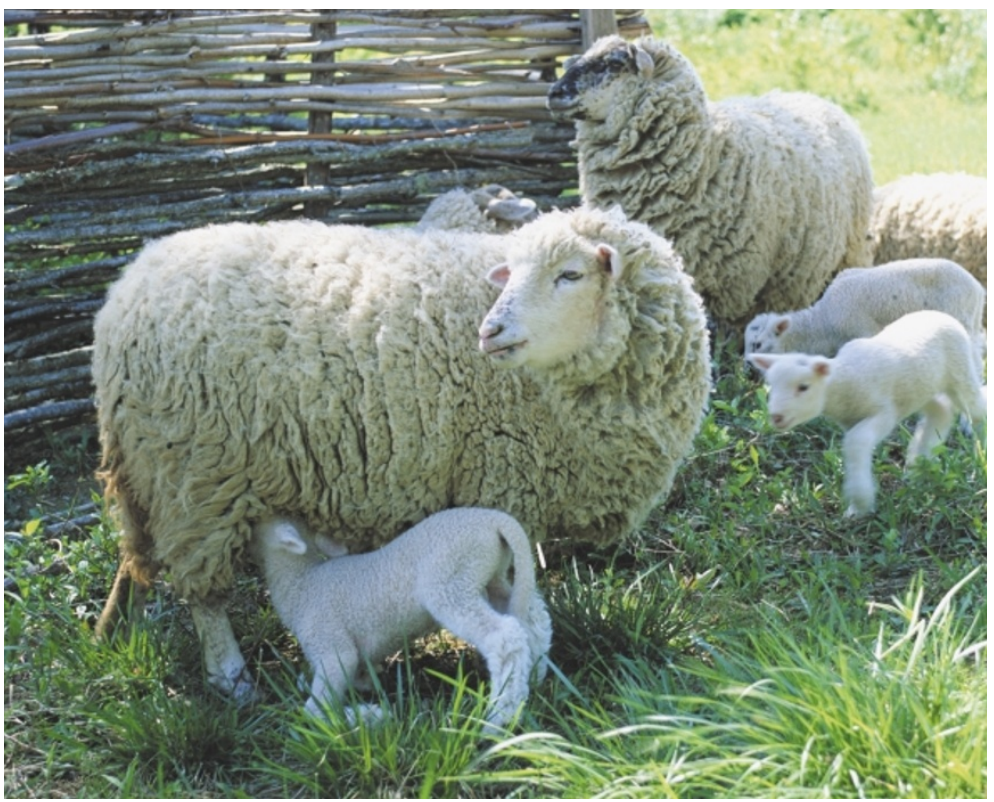

Cloning mammals has been riddled with problems: only a small proportion of cloned embryos survive to term and those that do are abnormally large, with respiratory and circulatory defects. This 'large offspring syndrome' has been thought to be due to the misregulation of imprinted genes, which are known to affect fetal growth. Now, Humpherys et al. confirm that imprinted genes are indeed misregulated in cloned mice, probably because of alterations in the methylation of their regulatory regions in ES cells before cloning.

Humpherys et al. began their study by comparing the expression levels of imprinted loci in embryonic and embryonically derived tissue from mice cloned by nuclear transfer with those of normal mice. Gene expression levels in wild-type embryos matched those of their placental controls, but expression levels in cloned embryos and their placentas varied significantly from each other and from the controls. These changes in expres- sion levels were found to occur independently at each locus.

To see if altered methylation states were the cause of this misregulation, the authors analysed the methylation of the upstream differentially methylated region (DMR) of two genes H19 and Igf2 - that were known to affect their expression. As expected, silenced loci had highly methylated DMRs; conversely, highly expressed loci were partially demethylated. As before, the methylation states of individual loci were independent of each other. Despite the wide variation in gene expression levels, fetal size or postnatal survival was not correlated with variation in the the expression of any single gene, indicating that these defects are probably a result of simultaneous changes in the expression of many genes.

But when does the reprogramming of epigenetic states occur? During nuclear cloning or in the donor ES cell populations? Humpherys et al. found that gene

\title{
Genome prospecting
}

Once you've identified the genes in a newly sequenced genome, the going gets tougher How do you find the less obvious functional elements - such as regulatory regions and the small non-translated genes? As discussed in Highlights last month, one approach is to search for known sequence motifs. But two more recent papers have shown how the tools of comparative and functional genomics can be used to tackle this challenge.

Mark Johnston and colleagues (Clifton $e t$ al.) investigated the power of comparative genomics for analysing the Saccharomyces cerevisiae genome. By generating over 4 $\mathrm{Mb}$ of genomic sequence from seven additional Saccharomyces species, they examined how the evolutionary divergence of the species influences the ability to identify short genomic sequences with specific functions. First, they sequenced and compared small nucleolar RNA (snoRNA) genes, which are conserved in all species. In pairwise comparisons between species that are too closely related, the genes did not stand out from the high background level of sequence conservation; conversely, the genomic sequences of species that were too diverged were difficult to align. Similar results were obtained for regulatory elements, but in this case it was valuable, particularly for the more diverged species, to align orthologous genes before searching for the upstream conserved elements. The authors conclude that the optimal strategy for sequence comparison with $S$. cerevisiae should involve at least three other species - two closely related species, such as $S$. mikatae and S. bayanus, and a more divergent species, such as $S$. kluyveri.

By combining comparative and functional genomics, Karen Wassarman, Francis Repoila and colleagues focused on finding new genes that encode small non-coding RNAs (sRNAs) in the Escherichia coli genome. They first compared the genome of $E$. coli with closely related Salmonella and Klebsiella species, concentrating on intergenic regions, which are known to contain sRNAs. They then used oligonucleotide microarrays and Northern blot analysis to test whether the sequences were expressed. Ultimately, they identified 17 new sRNAs ranging in size from 45 to 320 bases.

Wassarman et al. investigated the biological significance of these sRNAs by looking at the potential interaction with Hfq - an sRNA-binding protein that is involved in aspects of RNA metabolism, such as the regulation of translation, polyadenylation and mRNA stability. More than half of the newly discovered sRNAs co-immunoprecipitated with $\mathrm{Hfq}$ and are therefore implicated in gene regulation.

Both these studies show how bioinformatic and experimental approaches can be used to unearth the more elusive functional elements in genomes. Even in genomes as well studied as those of E. coli and S. cerevisiae, it is clear that genome prospectors have plenty more riches to discover.

Mark Patterson

(2) References and links ORIGINAL RESEARCH PAPERS Clifton, P. F. etal. Surveying Saccharomyces genomes to identify functional elements by comparative sequence analysis. Genome Res. 11, 1175-1186 (2001) | Wassarman, K. M. et al. Idenfitication of novel small RNAs using comparative genomics and microarrays. Genes Dev. 15, 1637-1651 (2001)

WEB SITES Mark Johnston's lab | Gisela Storz's lab | Susan Gottesman's lab 
expression levels and DMR methylation states varied greatly in all nine ES cell lines tested. Similar variation was observed even between subclones from a single ES cell line. These observations indicate that the epigenetic changes occur while ES cells are cultured, supporting previous suggestions that their epigenetic states are very unstable - so unstable that expression levels vary even between animals derived from the same ES cell subclones. Contrary to previous beliefs, mammalian development must be relatively robust because cloned embryos can survive despite such variation in gene expression.

It remains to be seen if human ES cells have similarly variable gene expression. If so, their clinical applications might be more limited than previously expected.

Magdalena Skipper

(2) References and links ORIGINAL RESEARCH PAPER Humpherys, D. et al. Epigenetic instability in ES cells and cloned mice. Science 293, 95-97 (2001) WEB SITE Rudolf Jaenisch's lab

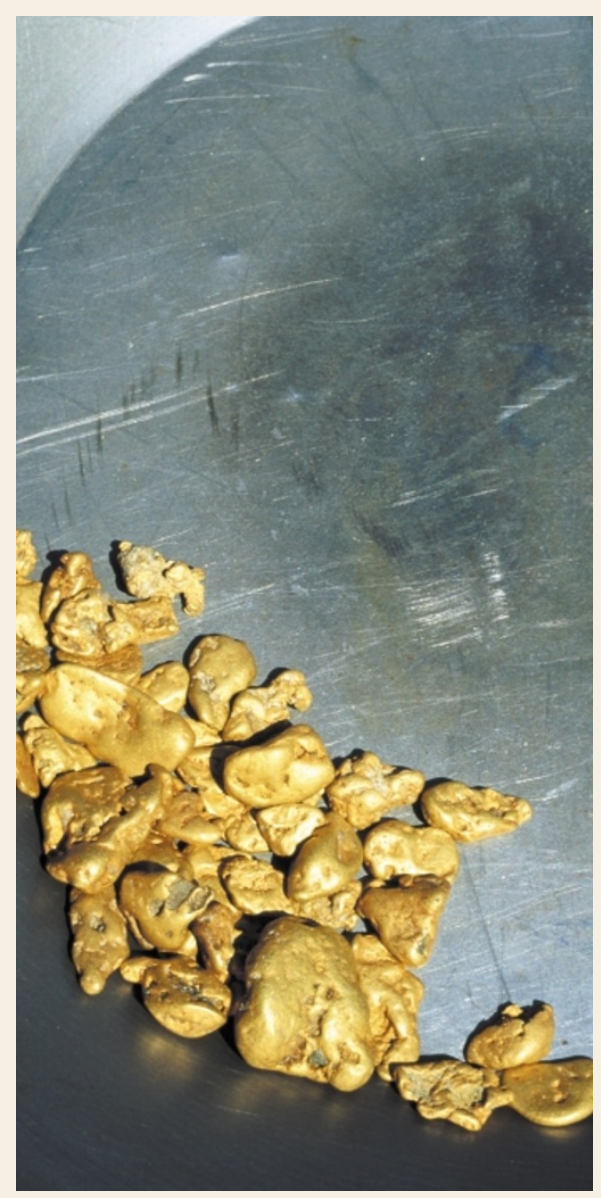

TECHNOLOGY

\section{A better mouse trap}

Loss-of-function mutants are available for only a small fraction of mouse genes, but this shortage is being addressed by several strategies, such as chemical mutagenesis, gene targeting and gene trapping. Insertion vectors are used as mutagens in gene trapping, which therefore has the advantage that mutated genes can be cloned rapidly from any mutant strain of interest. However, there has been some concern that gene trapping might lead to loss-offunction mutations only infrequently and that it is not efficient enough for large-scale projects. A recent paper by Bill Skarnes and colleagues seems to lay these concerns to rest.

Gene-trapping vectors contain reporter genes that are only expressed if the vector integrates within an expressed gene.

Transfected mouse ES cells that express the reporter gene are therefore likely to carry a gene interrupted by the vector, and the phenotypic consequences of this can then be studied in mouse embryos. The vector used by Mitchell et $a l$. was designed to express the reporter genes only if the vector was inserted into a gene that expresses a signal sequence or transmembrane domain. They reasoned that secreted or membrane-spanning proteins are likely to be involved in important developmental processes, such as signalling and cell adhesion, and that their disruption will cause a range of developmental phenotypes.

Over 500 ES cell lines that express the reporter genes were studied and most contained insertions in genes known, or predicted, to encode secreted or transmembrane proteins. From these lines, 60 were used to create mouse embryos, of which 25 had a clearly abnormal phenotype. The phenotypes ranged from early defects in processes such as gastrulation and implantation to abnormalities in later developing structures, such as the limb and eye. Furthermore, 11 of these mutants could be compared with existing gene knockouts, and in 10 cases the null phenotype was identical to that of the gene-trap mutant. This indicates that the secretory gene-trapping method efficiently generates loss-of-function mutations, and can be used in both gene-based and phenotypedriven screens. The authors argue that a closer analysis of the 35 lines that do not have overt abnormalities is also likely to uncover further

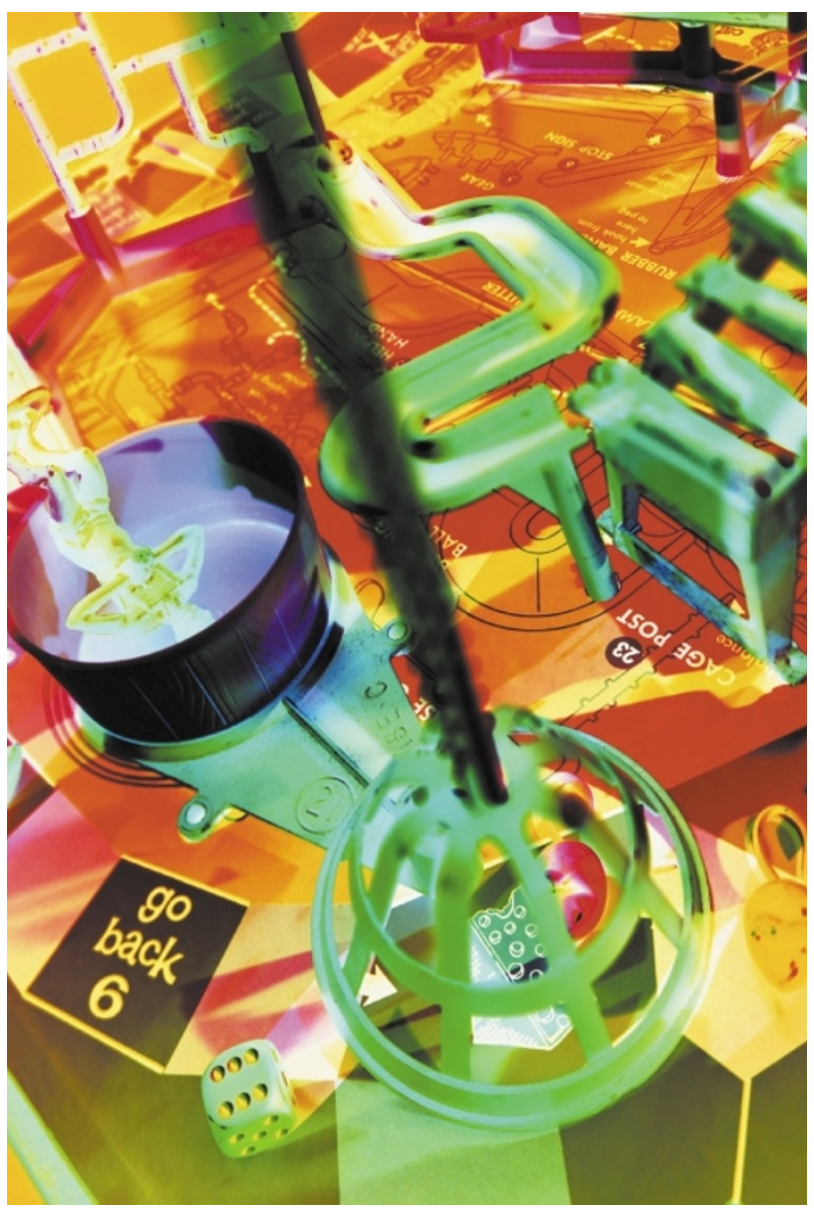

phenotypes. Indeed, a concurrent screen using a cell-autonomous axonal marker in the genetrap vector has revealed axon guidance defects in two of these lines.

Overall, Mitchell et al. can assign new functions to nine known, and five new, genes. In an accompanying News and Views, Ian Jackson likens those who argue about whether forward or reverse genetics is better for functional analysis of mouse genes to the Lilliputians and Blefuscans in Gulliver's Travels, who fought over which end of a boiled egg should be eaten first. By combining aspects of forward genetic screens and knockout technology, gene trappers might be said to be tackling the egg from somewhere in the middle. However, in this case the competition seems to be healthy and the technology for studying gene function is advancing on all fronts.

Mark Patterson

\section{(2) References and links}

ORIGINAL RESEARCH PAPER Mitchell, K. et al. Functional analysis of secreted and transmembrane proteins critical for mouse development. Nature Genet. 28, 241-249 (2001)

FURTHER READING Jackson, I. Mouse mutagenesis on target. Nature Genet. 28, 198-200 (2001) | Leighton, P. A. et al. Defining brain wiring patterns and mechanisms through gene trapping in mice. Nature 410, 174-179 (2001)

WEB SITE Bill Skarnes' lab 